\title{
Nutrition of children and gastrointestinal microflora in the insult of diagnostic parameters
}

\section{Abstract}

Does dietary intervention affect the change of the intestinal microflora, causes the unsealing of the intestinal mucosa. After four weeks of dietary intervention with FODMAP diets and a diet based on NICE recommendations in children with functional abdominal pain, the intestinal microflora will change and the level of zonulin will decrease.

Bacterial intestine, intestinal bacteria is a set of microorganisms, the main bacteria that form a complex ecosystem in the digestive tract. Scientific research indicates that some bacteria may contribute to the formation of certain disease entities.

Children with functional abdominal pain participated in the research.

The study was a randomized intervention and a double-blind test. 32 children were examined, patients of gastroenterological counseling center of the Provincial Specialist Children's Hospital in Olsztyn. Children were 4-12 years old, functional abdominal pain was diagnosed on the basis of Roman criteria, a positive decision of the gastroenterologist regarding the inclusion of the patient, conscious and voluntary consent of the child's guardian to participate in the study. Exclusion criteria include irritable bowel syndrome, abdominal migraine, diagnosed food allergies or food intolerance, the coexistence of chronic diseases or antibiotic therapy in the last 8 weeks.

The dietary intervention lasted 4 weeks, meals were prepared on the basis of a menu developed by a dietician.

Diagnostic tests were carried out before and after dietary intervention. One group of children with functional abdominal pain received the Fodmap diet, the second group, a diet based on NICE recommendations.

The intestinal microflora, the level of zonulin in the stool were tested and the organic acids in the urine were determined by capillary gas chromatography/mass spectrometers (GC/MS).

A preliminary analysis of test conditions prior to dietary intervention was found.

Dysbiosis occurred in all 26 children (4 children did not perform the test). An increased number of Bacteroides spp. Was observed in the majority of children in stool tests, studies have reported that the growth of this bacterium in the intestinal microflora occurs in IBD intestinal inflammatory syndrome. Preliminary researches show that this bacterium was dominant in the examined children. In the test, an intermediate urine dysbiosis test of 24 out of 26 children was tested had at least one parameter elevated. After the dietary intervention, the diet had an effect on changing the intestinal microflora, changing the parameters of dysbiosis and the parameter of zonulin, although more research is needed on a larger population.

\section{Conflict of Interest}

There is no conflict of interest 